Supplement of Atmos. Chem. Phys., 16, 15327-15345, 2016

http://www.atmos-chem-phys.net/16/15327/2016/

doi:10.5194/acp-16-15327-2016-supplement

(C) Author(s) 2016. CC Attribution 3.0 License.

(c) (i)

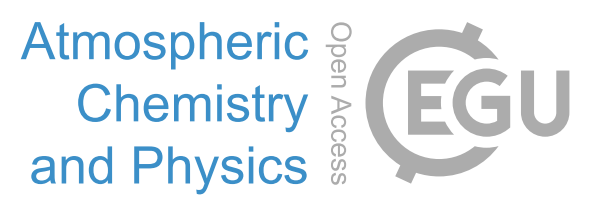

Supplement of

\title{
Evaluation of gas-particle partitioning in a regional air quality model for organic pollutants
}

Christos I. Efstathiou et al.

Correspondence to: Christos I. Efstathiou (efstathiou@ recetox.muni.cz)

The copyright of individual parts of the supplement might differ from the CC-BY 3.0 licence. 


\section{Contents}

Table S1. Summary of atmospheric processes and other features incorporated in recent regional scale modelling studies of POPs.

Table S2. Detailed information about the BaP concentration and deposition measurement stations operated by EMEP during 2006.

Figure S3. Annual average total elemental carbon (EC) concentrations $\left(\mu \mathrm{g} \mathrm{m}^{-3}\right)$ calculated at the level of the surface layer by WRF-CMAQ-BaP during 2006.

Figure S4. Annual average ozone concentrations (ppmV) calculated at the level of the surface layer by WRF-CMAQ-BaP during 2006.

Figure S5. Seasonal differences in average surface-level $\mathrm{BaP}$ concentrations simulated by the dual GPP model (scenario 4) between the typical $\mathrm{K}_{\mathrm{oa}}$ estimates based on $\mathrm{K}_{\text {ow }}$ (Beyer, 2000), minus the semi-logarithmic temperature-dependent form proposed by Odabasi (2006).

Figure S6. Wintertime differences (Method B - Method A) in average surface-level $\mathrm{BaP}$ concentrations simulated by the dual GPP model (scenario 4) using two different methods for estimating the soot-air partitioning coefficient $\mathrm{K}_{\mathrm{SA}}$ (Method A: Dachs et al., 2000; Method B: van Noort, 2008).

Figure S7. Annual average BaP emission fluxes ( $\mathrm{g} / \mathrm{s}$ ) in the model cells (a) enclosing EMEP monitoring site locations, and (b) selected metropolitan areas of Europe during 2006.

Figure S8. Taylor diagram showing how three complementary model performance statistics (correlation coefficient, standard deviation, and the centred root-meansquare error) vary simultaneously for each one of the 2006 WRF-CMAQ-BaP simulation scenarios against EMEP measurements.

Table S9. Comparison between modelled and measured BaP concentrations for the entire 2006 EMEP dataset along with performance metrics and ranking for each individual site.

Figure S10. Modelled annual average (a) wet and (b) dry deposition ( $\mathrm{kg} / \mathrm{hectare})$ of BaP during 2006. 
2

\begin{tabular}{|c|c|c|c|c|c|c|c|c|}
\hline Reference & Galarneau $^{1}$ & Silibello $^{2}$ & Aulinger ${ }^{3}$ & San Jose ${ }^{4}$ & Inomata $^{5}$ & $\begin{array}{c}\text { Matthias }^{6} \\
\text { Bieser }^{9}\end{array}$ & Meng $^{7}$ & Cooter $^{8}$ \\
\hline Model Name & AURAMS & FARM & CMAQ & CMAQ & RAQM & CMAQ & CMAQ & CMAQ \\
\hline Domain & $\begin{array}{l}\text { USA \& } \\
\text { Canada }\end{array}$ & Italy & Europe & Spain & N.E. Asia & Europe & USA & NE USA \\
\hline Compound(s) & PAHs (BaP) & $\mathrm{BaP}$ & $\mathrm{BaP}$ & $\mathrm{BaP}$ & PAHs (BaP) & $\mathrm{BaP}$ & $\begin{array}{c}\mathrm{PCBs} \\
\mathrm{PCDD} / \mathrm{Fs}\end{array}$ & Atrazine \\
\hline Gas-phase reaction & Yes (Off-line) & Yes (SAPRC99) & Yes (CB4) & No (inert) & Yes & Yes (CB4) & Yes (CB4) & Yes (CB4) \\
\hline Adsorption model (J-P) & Yes & Yes & Yes & Yes & Yes & Yes & Yes & Yes \\
\hline Aerosol water dissolution & No & Yes & Yes & No & No & Yes & No & Yes \\
\hline Absorption to OM & Yes & Yes & Yes & No & No & Yes & Yes & Yes \\
\hline Adsorption to EC/BC & Yes & No & No & No & No & No & No & No \\
\hline $\begin{array}{l}\text { Heterogeneous chemistry } \\
\text { (e.g. } \mathrm{O}_{3} \text { reaction) }\end{array}$ & No & No & No & Yes & No & Yes & No & No \\
\hline
\end{tabular}

${ }^{1}$ Galarneau et al., 2014; ${ }^{2}$ Silibello et al., 2012; ${ }^{3}$ Aulinger et al., 2007; ${ }^{4}$ San José et al., 2013; ${ }^{5}$ Inomata et al., $2012 ;{ }^{6}$ Matthias et al., 2009; ${ }^{7}$ Meng et al., 2007; ${ }^{8}$ Cooter and Hutzell, 2002; ${ }^{9}$ Bieser et al., 2012 
Table S2. Detailed information about the BaP concentration and deposition measurement stations operated by EMEP during 2006.

\begin{tabular}{|c|c|c|c|c|c|c|c|}
\hline Code & Country & Station & $\begin{array}{l}\text { Latitude } \\
\left({ }^{\circ} \mathrm{N}\right)\end{array}$ & $\begin{array}{l}\text { Longitu } \\
\text { de }\left({ }^{\circ} \mathrm{E}\right)\end{array}$ & $\begin{array}{l}\text { Altitu } \\
\text { de }(m)\end{array}$ & $\begin{array}{l}\text { Concentration } \\
\text { sampling }\end{array}$ & Deposition sampling \\
\hline CZ0003R & $\begin{array}{l}\text { Czech } \\
\text { Republic }\end{array}$ & Kosetice & 49.58 & 15.08 & 534 & $\begin{array}{l}1 \text { day per week, } \\
\text { air+aerosol }\end{array}$ & $\begin{array}{l}1 \text { day per week, wet } \\
\text { deposition }\end{array}$ \\
\hline ES0008R & Spain & Niembro & 43.44 & -4.85 & 134 & $\begin{array}{l}1 \text { day per week, } \\
\text { air+aerosol }\end{array}$ & NA \\
\hline FI0036R & Finland & Pallas & 68 & 24.24 & 340 & $\begin{array}{l}1 \text { week per month, } \\
\text { air+aerosol }\end{array}$ & $\begin{array}{l}1 \text { week per month, } \\
\text { precip+dry } \\
\text { deposition }\end{array}$ \\
\hline LV0010R & Latvia & Rucava & 56.16 & 21.17 & 18 & Monthly, aerosol & NA \\
\hline LV0016R & Latvia & Zoseni & 57.14 & 25.91 & 188 & Monthly, aerosol & NA \\
\hline SE0012R & Sweden & Aspvreten & 58.8 & 17.38 & 20 & $\begin{array}{l}1 \text { week per month, } \\
\text { air+aerosol }\end{array}$ & $\begin{array}{l}\text { Monthly (4wk), } \\
\text { precip+dry } \\
\text { deposition }\end{array}$ \\
\hline SE0014R & Sweden & Råö & 57.39 & 11.91 & 5 & $\begin{array}{l}2 \text { week per month, } \\
\text { air+aerosol }\end{array}$ & $\begin{array}{l}\text { Monthly (4wk), } \\
\text { precip+dry } \\
\text { deposition }\end{array}$ \\
\hline GB0014R & U.K. & High Muffles & 54.33 & -0.81 & 267 & $\begin{array}{l}3 \text { months per yr, } \\
\text { ads_tube }\end{array}$ & NA \\
\hline DE0009R & Germany & Zingst & 54.43 & 12.73 & 1 & NA & $\begin{array}{l}\text { Monthly ( } 4 w k) \text {, wet } \\
\text { deposition }\end{array}$ \\
\hline DE0001R & Germany & Westerland & 54.93 & 8.31 & 12 & NA & $\begin{array}{l}\text { Monthly (4wk), wet } \\
\text { deposition }\end{array}$ \\
\hline
\end{tabular}




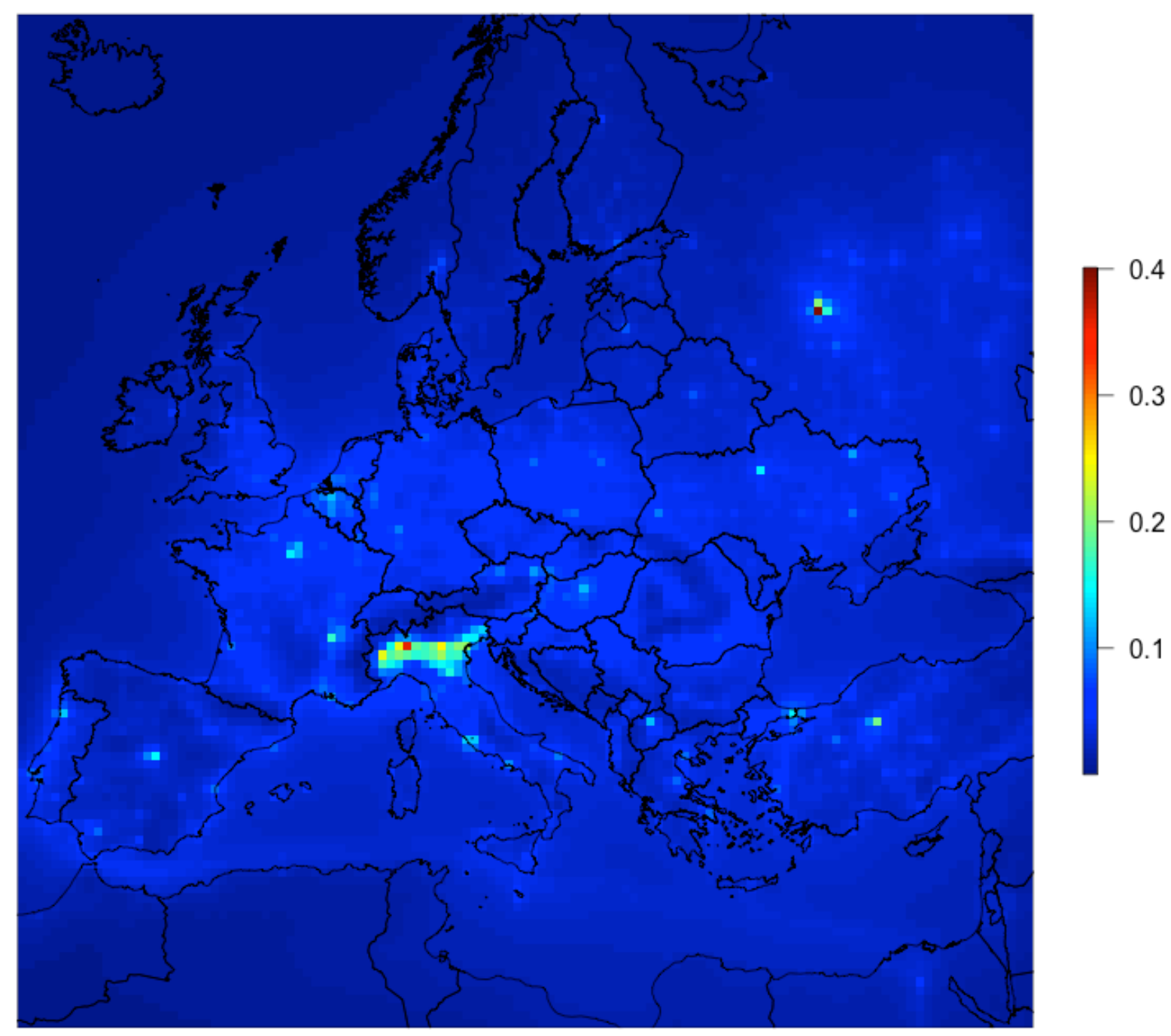

Figure S3. Annual average total elemental carbon (EC) concentrations $\left(\mu \mathrm{g} \mathrm{m}^{-3}\right)$ calculated at the level of the surface layer by WRF-CMAQ-BaP during 2006. 


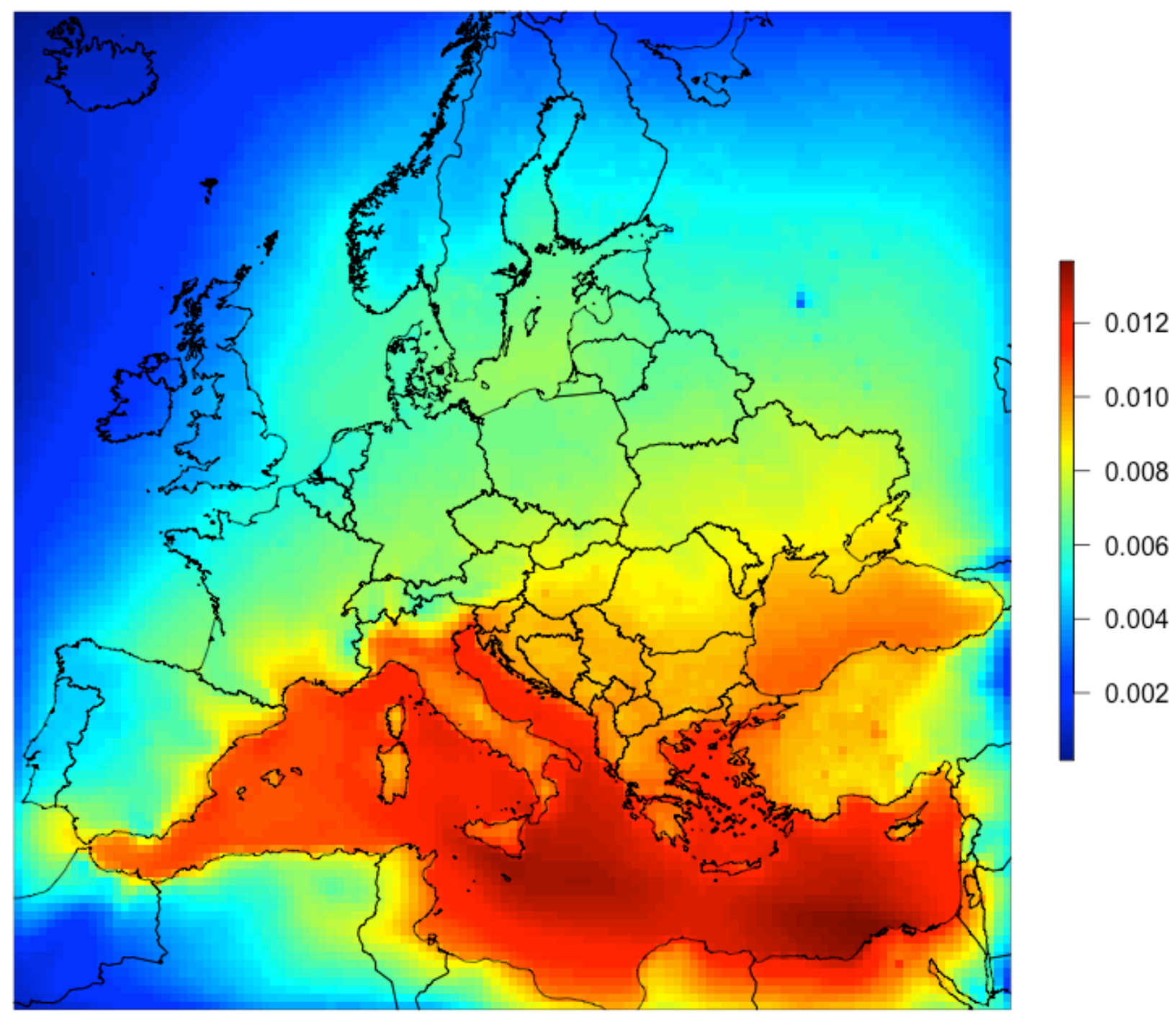

Figure S4. Annual average ozone concentrations ( $\mathrm{ppmV}$ ) calculated at the level of the surface layer by WRF-CMAQ-BaP during 2006. 


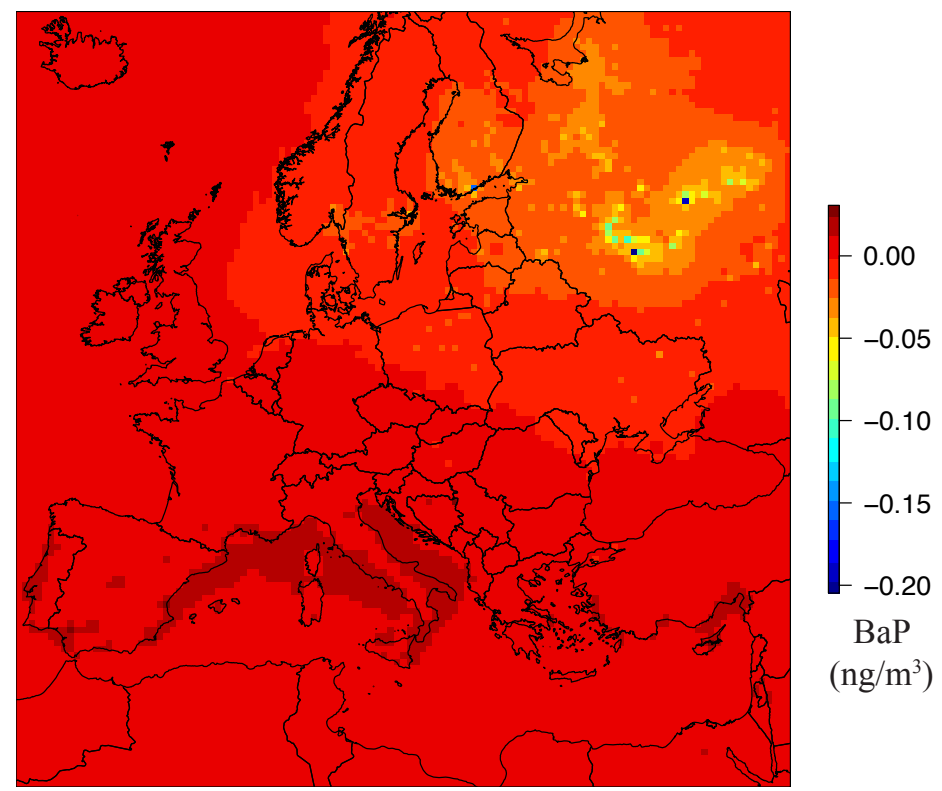

Winter

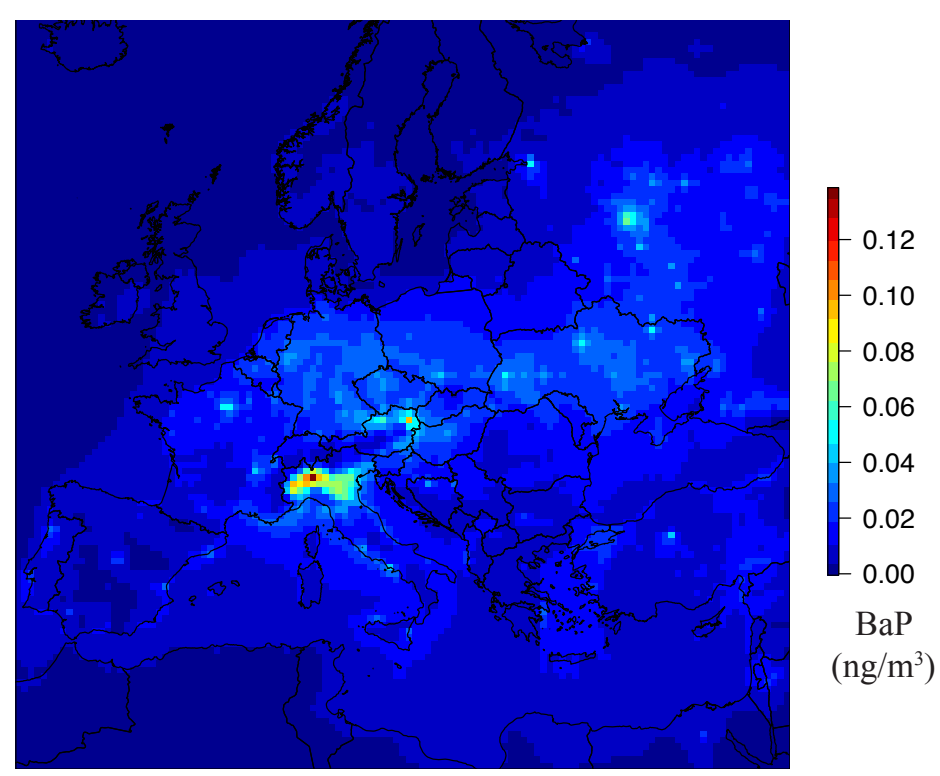

Summer

Figure S5. Seasonal differences in average surface-level BaP concentrations simulated by the dual GPP model (scenario 4) 5 between the typical $\mathrm{K}_{\text {oa }}$ estimates based on $\mathrm{K}_{\text {ow }}$ (Beyer, 2000), minus the semi-logarithmic temperature-dependent form proposed by Odabasi (2006). 


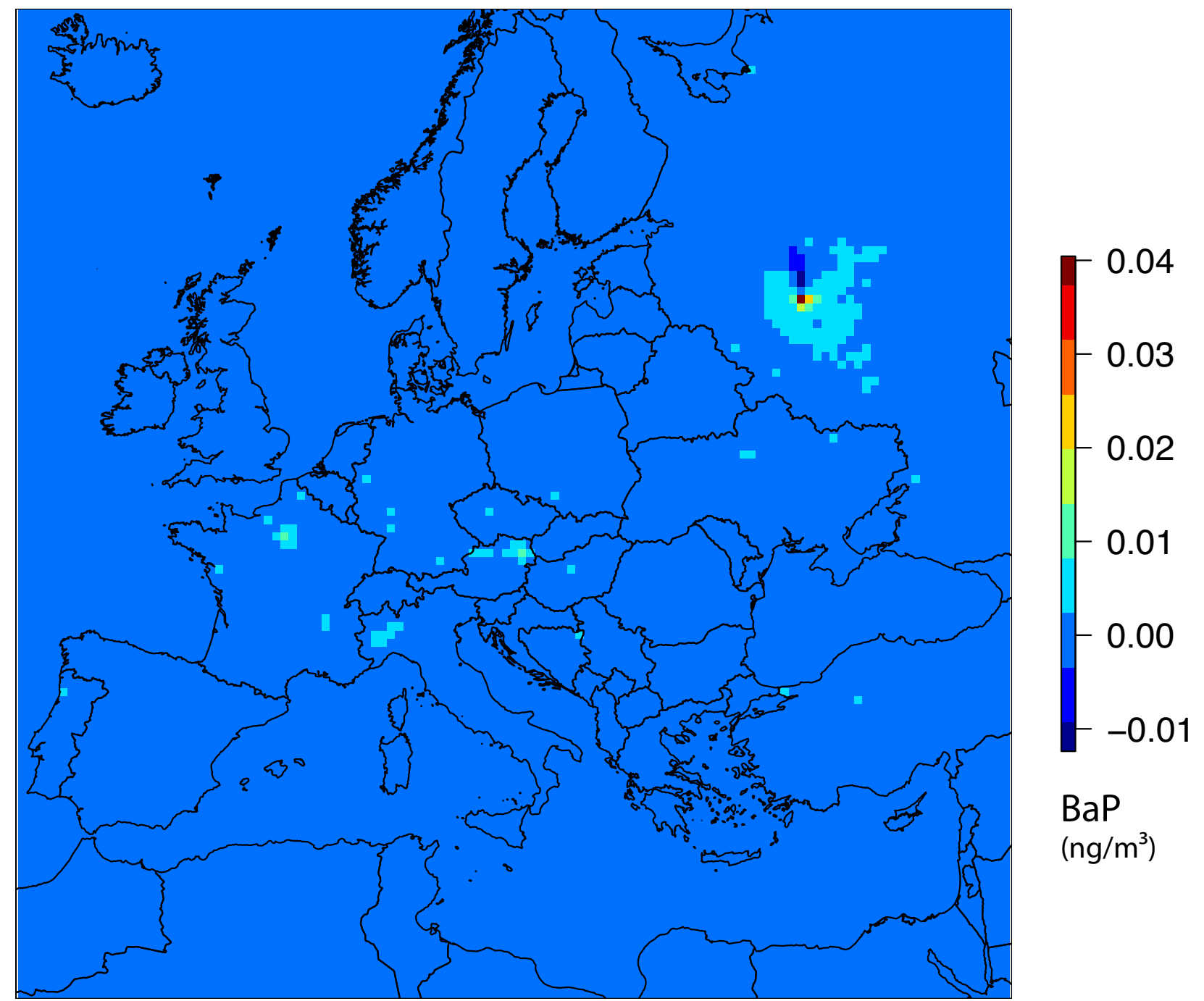

Figure S6. Wintertime differences (Method B - Method A) in average surface-level BaP concentrations simulated by the dual GPP model (scenario 4) using two different methods for estimating the soot-air partitioning coefficient $\mathrm{K}_{\mathrm{SA}}$ (Method A: Dachs et al., 2000; Method B: van Noort, 2008). 


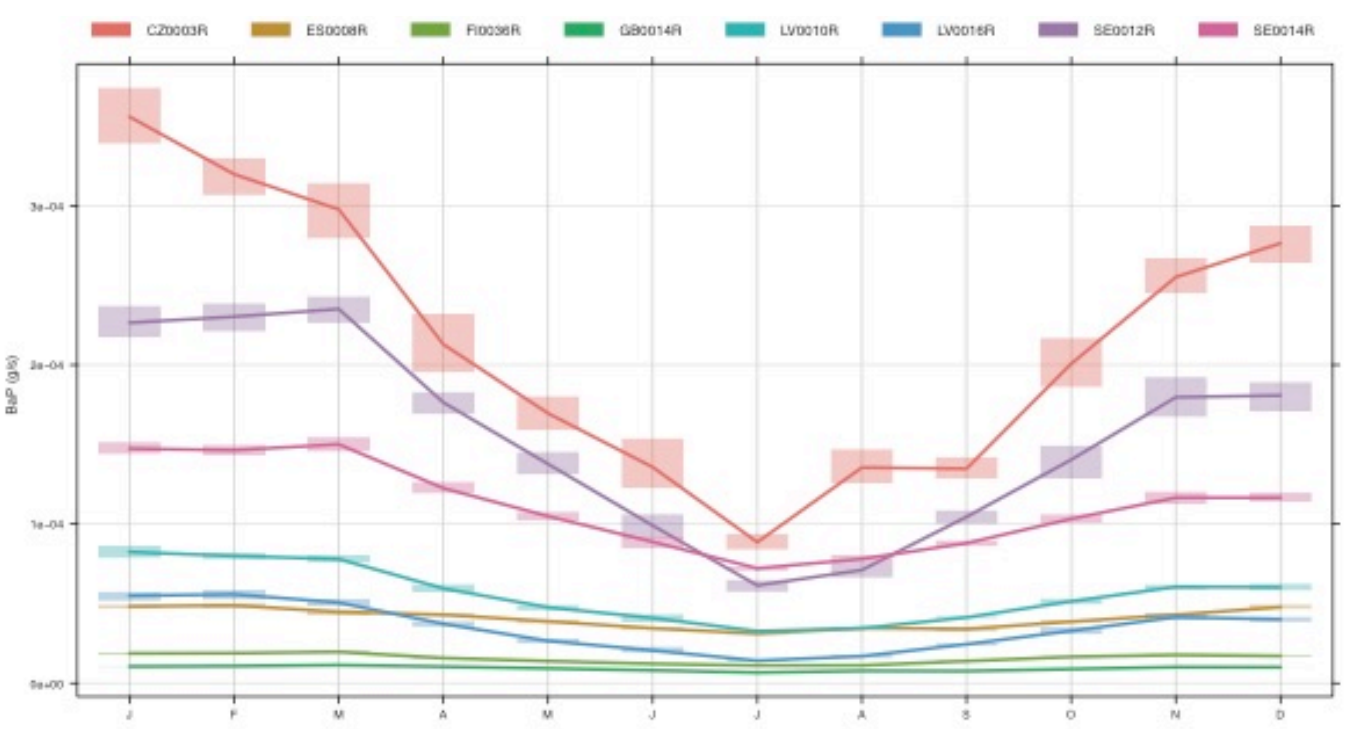

(a)

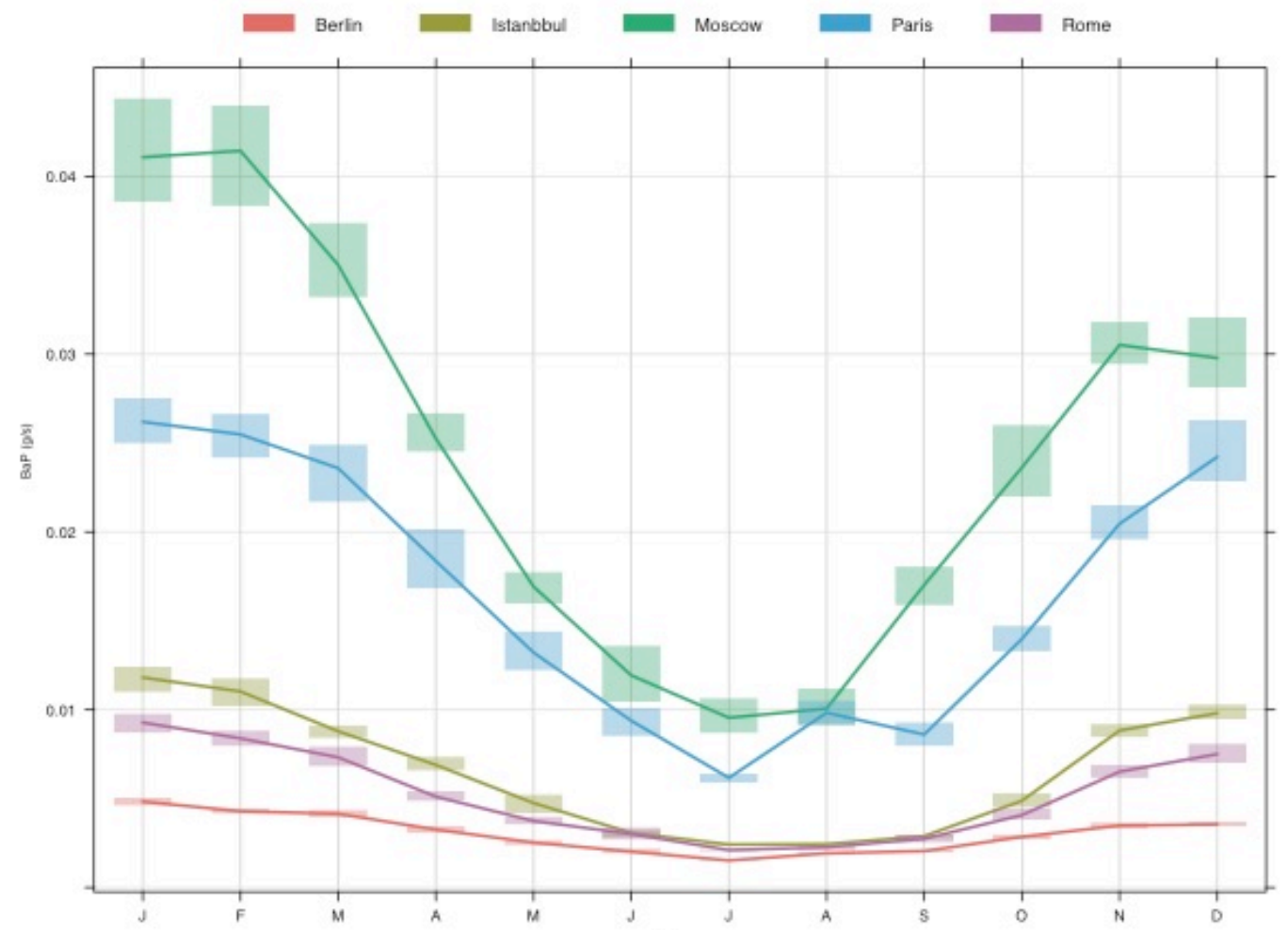

(b)

Figure S7. Annual average BaP emission fluxes ( $\mathrm{g} / \mathrm{s}$ ) in the model cells (a) enclosing EMEP monitoring site locations, and (b) selected metropolitan areas of Europe during 2006. 


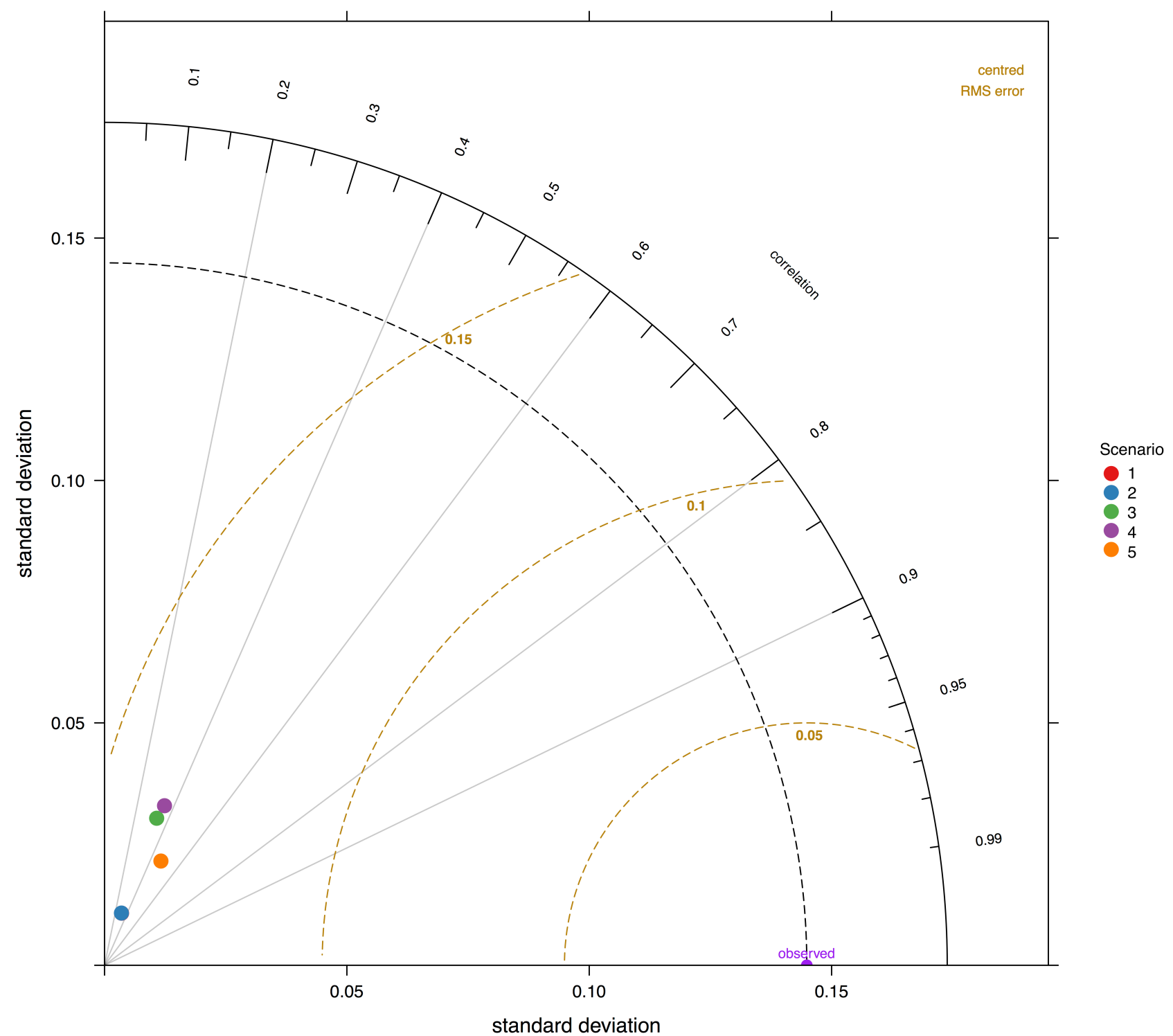

Figure S8. Taylor diagram showing how three complementary model performance statistics (correlation coefficient, standard deviation, and the centred root-mean-square error) vary simultaneously for each one of the 2006 WRF-CMAQ-BaP 5 simulation scenarios against EMEP measurements. 
Table S9. Comparison between modelled and measured BaP concentrations for the entire 2006 EMEP dataset along with performance metrics and ranking for each individual site.

\begin{tabular}{|c|c|c|c|c|c|c|c|c|c|c|}
\hline \multicolumn{11}{|c|}{$\overline{C Z 0003 R ~(n=49) ~}$} \\
\hline Rank & Scenario & FAC2 & $\mathrm{MB}$ & MGE & NMB & NMGE & RMSE & $\mathrm{r}$ & $\mathrm{COE}$ & IOA \\
\hline$\overline{1}$ & 5 & 0.36735 & -0.08637 & 0.09609 & -0.70689 & 0.78640 & 0.19376 & 0.54963 & 0.32679 & 0.66339 \\
\hline 2 & 3 & 0.36735 & -0.07212 & 0.10288 & -0.59027 & 0.84202 & 0.19932 & 0.23115 & 0.27917 & 0.63958 \\
\hline 3 & 4 & 0.32653 & -0.05801 & 0.10339 & -0.47476 & 0.84617 & 0.19225 & 0.28326 & 0.27562 & 0.63781 \\
\hline 4 & 1 & 0.44898 & -0.09848 & 0.10690 & -0.80599 & 0.87492 & 0.21169 & 0.32111 & 0.25101 & 0.62550 \\
\hline 5 & 2 & 0.44898 & -0.09848 & 0.10690 & -0.80601 & 0.87493 & 0.21169 & 0.32112 & 0.25100 & 0.62550 \\
\hline \multicolumn{11}{|c|}{ ES0008R (n=49) } \\
\hline Rank & Scenario & FAC2 & $\mathrm{MB}$ & MGE & NMB & NMGE & RMSE & $\mathrm{r}$ & $\mathrm{COE}$ & IOA \\
\hline 1 & 4 & 0.26531 & -0.02635 & 0.02964 & -0.69933 & 0.78686 & 0.04848 & -0.02821 & -0.27066 & 0.36467 \\
\hline 2 & 3 & 0.08163 & -0.03105 & 0.03232 & -0.82423 & 0.85797 & 0.05036 & -0.00232 & -0.38549 & 0.30726 \\
\hline 3 & 5 & 0.06122 & -0.03357 & 0.03369 & -0.89104 & 0.89434 & 0.05158 & -0.04224 & -0.44422 & 0.27789 \\
\hline 4 & 1 & 0.00000 & -0.03446 & 0.03446 & -0.91481 & 0.91481 & 0.05190 & 0.03887 & -0.47727 & 0.26136 \\
\hline 5 & 2 & 0.00000 & -0.03449 & 0.03449 & -0.91550 & 0.91550 & 0.05192 & 0.02794 & -0.47839 & 0.26081 \\
\hline \multicolumn{11}{|c|}{$\overline{\text { FI0036R }(n=12)}$} \\
\hline Rank & Scenario & FAC2 & $\mathrm{MB}$ & MGE & NMB & NMGE & RMSE & $\mathrm{r}$ & $\mathrm{COE}$ & IOA \\
\hline 1 & 4 & 0.50000 & -0.01179 & 0.01358 & -0.72184 & 0.83163 & 0.03614 & 0.89444 & 0.36985 & 0.68492 \\
\hline 2 & 3 & 0.41667 & -0.01268 & 0.01373 & -0.77616 & 0.84042 & 0.03627 & 0.90937 & 0.36319 & 0.68159 \\
\hline 3 & 5 & 0.33333 & -0.01427 & 0.01473 & -0.87360 & 0.90210 & 0.03984 & 0.38981 & 0.31645 & 0.65823 \\
\hline 4 & 1 & 0.16667 & -0.01560 & 0.01560 & -0.95507 & 0.95507 & 0.04070 & 0.45114 & 0.27631 & 0.63815 \\
\hline 5 & 2 & 0.08333 & -0.01563 & 0.01563 & -0.95708 & 0.95708 & 0.04072 & 0.42418 & 0.27479 & 0.63739 \\
\hline \multicolumn{11}{|c|}{ LV0010R $(n=11)$} \\
\hline Rank & Scenario & FAC2 & MB & MGE & NMB & NMGE & RMSE & $\mathrm{r}$ & $\mathrm{COE}$ & IOA \\
\hline 1 & 4 & 0.00000 & -0.27764 & 0.27764 & -0.92828 & 0.92828 & 0.36661 & 0.80956 & -0.32366 & 0.33817 \\
\hline 2 & 3 & 0.00000 & -0.28174 & 0.28174 & -0.94198 & 0.94198 & 0.36970 & 0.84842 & -0.34319 & 0.32841 \\
\hline 3 & 5 & 0.00000 & -0.28967 & 0.28967 & -0.96852 & 0.96852 & 0.37933 & 0.51157 & -0.38103 & 0.30948 \\
\hline 4 & 1 & 0.00000 & -0.29504 & 0.29504 & -0.98646 & 0.98646 & 0.38476 & 0.85236 & -0.40662 & 0.29669 \\
\hline 5 & 2 & 0.00000 & -0.29527 & 0.29527 & -0.98722 & 0.98722 & 0.38496 & 0.85706 & -0.40770 & 0.29615 \\
\hline
\end{tabular}

Continued on next page 
Table S8 Continued

\begin{tabular}{|c|c|c|c|c|c|c|c|c|c|c|}
\hline \multicolumn{11}{|c|}{ LV0016R $(n=12)$} \\
\hline \multirow{2}{*}{$\frac{\text { Rank }}{1}$} & \multicolumn{2}{|c|}{ Scenario FAC2 } & \multirow{2}{*}{$\begin{array}{l}\mathrm{MB} \\
-0.07158\end{array}$} & \multirow{2}{*}{$\begin{array}{l}\text { MGE } \\
0.07227\end{array}$} & \multirow{2}{*}{$\begin{array}{l}\text { NMB } \\
-0.72059\end{array}$} & \multirow{2}{*}{$\begin{array}{l}\text { NMGE } \\
0.72752\end{array}$} & \multirow{2}{*}{$\begin{array}{l}\text { RMSE } \\
0.09057\end{array}$} & \multirow{2}{*}{$\begin{array}{l}\mathrm{r} \\
0.88148\end{array}$} & \multirow{2}{*}{$\begin{array}{l}\text { COE } \\
-0.33827\end{array}$} & \multirow{2}{*}{$\begin{array}{l}\text { IOA } \\
0.33086\end{array}$} \\
\hline & 4 & 0.08333 & & & & & & & & \\
\hline 2 & 3 & 0.08333 & -0.07546 & 0.07570 & -0.75964 & 0.76204 & 0.09297 & 0.88465 & -0.40177 & 0.29911 \\
\hline 3 & 5 & 0.08333 & -0.08749 & 0.08749 & -0.88077 & 0.88077 & 0.10770 & 0.84240 & -0.62019 & 0.18991 \\
\hline 4 & 1 & 0.08333 & -0.09485 & 0.09485 & -0.95487 & 0.95487 & 0.11846 & 0.87319 & -0.75649 & 0.12176 \\
\hline 5 & 2 & 0.08333 & -0.09488 & 0.09488 & -0.95517 & 0.95517 & 0.11850 & 0.87173 & -0.75705 & 0.12148 \\
\hline \multicolumn{11}{|c|}{ SE0012R $(n=12)$} \\
\hline Rank & \multicolumn{2}{|c|}{ Scenario FAC2 } & MB & MGE & NMB & NMGE & RMSE & $\mathrm{r}$ & $\mathrm{COE}$ & IOA \\
\hline 1 & 4 & 0.50000 & -0.05197 & 0.05303 & -0.71114 & 0.72557 & 0.08451 & 0.79238 & 0.22525 & 0.61262 \\
\hline 2 & 3 & 0.50000 & -0.05462 & 0.05490 & -0.74732 & 0.75119 & 0.08590 & 0.78000 & 0.19790 & 0.59895 \\
\hline 3 & 5 & 0.25000 & -0.05926 & 0.05940 & -0.81079 & 0.81274 & 0.09213 & 0.72644 & 0.13217 & 0.56609 \\
\hline 4 & 1 & 0.00000 & -0.06656 & 0.06656 & -0.91074 & 0.91074 & 0.09943 & 0.69886 & 0.02753 & 0.51377 \\
\hline 5 & 2 & 0.00000 & -0.06675 & 0.06675 & -0.91333 & 0.91333 & 0.09972 & 0.68275 & 0.02477 & 0.51238 \\
\hline \multicolumn{11}{|c|}{ SE0014R $(n=24)$} \\
\hline Rank & \multicolumn{2}{|c|}{ Scenario FAC2 } & $\mathrm{MB}$ & MGE & NMB & NMGE & RMSE & $\mathrm{r}$ & $\mathrm{COE}$ & IOA \\
\hline 1 & 4 & 0.33333 & -0.03390 & 0.04240 & -0.59250 & 0.74120 & 0.07728 & 0.75338 & 0.25318 & 0.62659 \\
\hline 2 & 3 & 0.37500 & -0.03674 & 0.04275 & -0.64223 & 0.74734 & 0.07807 & 0.78464 & 0.24699 & 0.62349 \\
\hline 3 & 5 & 0.33333 & -0.04268 & 0.04564 & -0.74610 & 0.79780 & 0.08329 & 0.72657 & 0.19615 & 0.59807 \\
\hline 4 & 1 & 0.29167 & -0.05009 & 0.05066 & -0.87565 & 0.88547 & 0.09049 & 0.67532 & 0.10782 & 0.55391 \\
\hline 5 & 2 & 0.29167 & -0.05042 & 0.05089 & -0.88133 & 0.88948 & 0.09073 & 0.68208 & 0.10378 & 0.55189 \\
\hline \multicolumn{11}{|c|}{ GB0014R $(n=4)$} \\
\hline Rank & \multicolumn{2}{|c|}{ Scenario FAC2 } & MB & MGE & NMB & NMGE & RMSE & $\mathrm{r}$ & $\mathrm{COE}$ & IOA \\
\hline 1 & 4 & 0.25000 & -0.02517 & 0.02613 & -0.71395 & 0.74117 & 0.03611 & 0.99549 & -0.10005 & 0.44997 \\
\hline 2 & 3 & 0.25000 & -0.02812 & 0.02812 & -0.79784 & 0.79784 & 0.03768 & 0.98569 & -0.18416 & 0.40792 \\
\hline 3 & 5 & 0.00000 & -0.03169 & 0.03169 & -0.89900 & 0.89900 & 0.04143 & 0.95280 & -0.33431 & 0.33285 \\
\hline 4 & 1 & 0.00000 & -0.03296 & 0.03296 & -0.93504 & 0.93504 & 0.04339 & 0.29286 & -0.38780 & 0.30610 \\
\hline 5 & 2 & 0.00000 & -0.03297 & 0.03297 & -0.93543 & 0.93543 & 0.04341 & 0.27833 & -0.38837 & 0.30581 \\
\hline
\end{tabular}




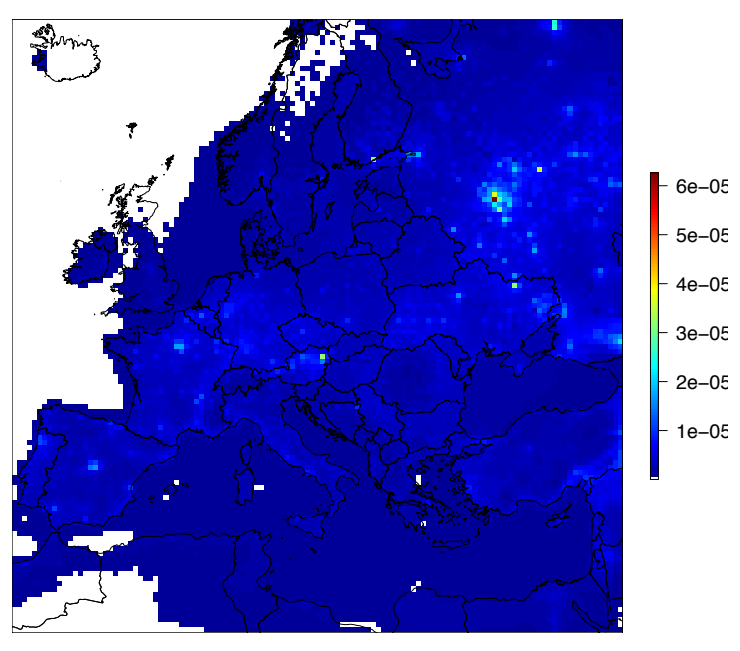

(a)

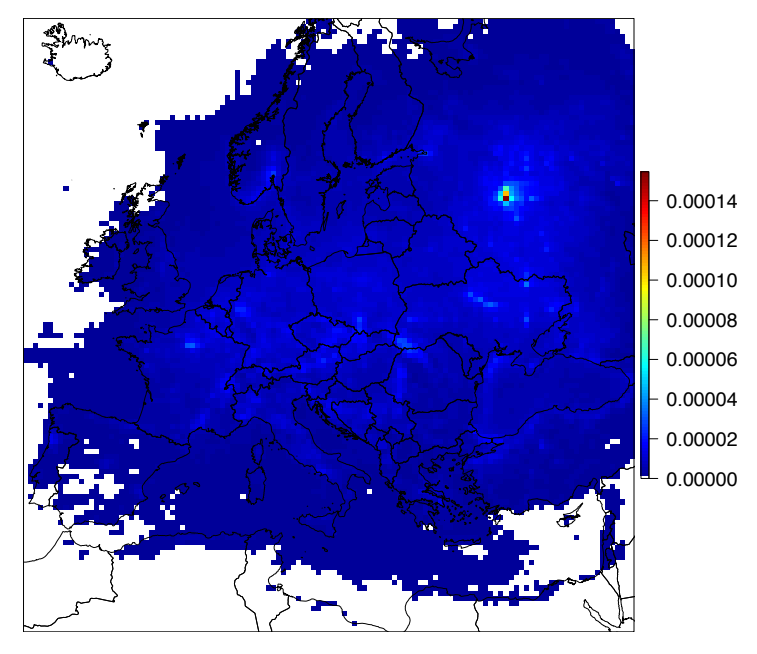

(b)

Figure S10. Modelled annual average (a) wet and (b) dry deposition (kg/hectare) of BaP during 2006. 